\title{
The Quantum Hall Effect in Graphene: Emergent Modular Symmetry and the Semi-circle Law
}

\author{
C.P. Burgess ${ }^{1,2}$ and Brian P. Dolan ${ }^{3,4}$ \\ 1 Department of Physics and Astronomy, McMaster University, \\ 1280 Main Street West, Hamilton, Ontario, Canada, L8S 4M1. \\ 2 Perimeter Institute, 31 Caroline Street North, Waterloo, Ontario, Canada. \\ 3 Dept. of Mathematical Physics, National University of Ireland, Maynooth, Ireland. \\ ${ }^{4}$ School of Theoretical Physics, Dublin Institute for Advanced Studies, 10 Burlington Rd., Dublin, Ireland. \\ e-mail: cburgess@perimeterinstitute.ca, bdolan@thphys.nuim.ie
}

(Dated: 11th December 2006)

\begin{abstract}
Low-energy transport measurements in Quantum Hall systems have been argued to be governed by emergent modular symmetries whose predictions are robust against many of the detailed microscopic dynamics. We propose the recently-observed quantum Hall effect in graphene as a test of these ideas, and identify to this end a class of predictions for graphene which would follow from the same modular arguments. We are led to a suite of predictions for high mobility samples that differs from those obtained for the conventional quantum Hall effect in semiconductors, including: predictions for the locations of the quantum Hall plateaux; predictions for the positions of critical points on transitions between plateaux; a selection rule for which plateaux can be connected by lowtemperature transitions; and a semi-circle law for conductivities traversed during these transitions. Many of these predictions appear to provide a good description of graphene measurements performed with intermediate-strength magnetic fields.
\end{abstract}

PACS numbers: 73.43.-f, 05.30.Fk, 02.20.-a

Recent quantum Hall experiments in single graphene layers [1, 2] have produced fascinating results which are markedly different from the now standard quantum Hall effect in semiconducting monolayers. In this note we point out that these experimental results, at least for intermediate values of the external magnetic field, are compatible with a suggestion, first made in 1989 [3], that a particular level 2 sub-group of the modular group might be a symmetry of the quantum Hall effect. Our main purpose is to identify the experimental implications of this symmetry, including the prediction that graphene should be expected to exhibit a semi-circle law, and to encourage experimentalists to look for these.

The idea that the modular group might be relevant to the quantum Hall effect in semiconducting heterostructures was explored in more detail in [4] where the existence of a group of symmetries was postulated, acting on the complex conductivity $\sigma=\sigma_{x y}+i \sigma_{x x}$ according to

$$
\sigma \rightarrow \frac{a \sigma+b}{c \sigma+d}
$$

where the integers $a$ through $d$ satisfy the constraint $a d-b c=1$. This defines the modular group, $\Gamma(1) \approx$ $S l(2 ; \mathbf{Z}) / \mathbf{Z}_{2}$. These authors argued that phase structure in the $\sigma$-plane for high-mobility samples was consistent with the existence of a symmetry of the above form (at least for samples with electron spins well-split). Further progress was made in [5, 6] where it was noticed that odd denominator states are singled out provided the integer $c$ is restricted to be even, defining the level-2 subgroup,
$\Gamma_{0}(2) \subset \Gamma(1)$. This subgroup differs from the subgroup $\Gamma_{\theta} \subset \Gamma(1)$ examined in ref. [3], which can be defined by the condition that the integers $b$ and $c$ both be even, or both be odd. In particular $\sigma \rightarrow-1 / \sigma$ is in $\Gamma_{\theta}$ and this is not a symmetry of the usual quantum Hall effect, there is no evidence in the experimental data for a fixed point at $\sigma=i$.

Similar conclusions were reached at much the same time in 7], where more detailed thinking about the microscopic dynamics led to the Law of Corresponding States, whose action on filling fractions implies an action on conductivities which agrees with $\Gamma_{0}(2)$ symmetry. Modular transformations, restricted to the real axis $\sigma_{x x}=0$, generate a $\Gamma_{0}(2)$ action on ground state wavefunctions, which is also implicit in the work of Jain and collaborators [8].

A puzzle which these considerations left open was the remarkable robustness of their experimental implications, which are found to apply well beyond the expected domain of validity of the microscopic derivation 9]. This robustness was clarified by subsequent work [10, 11, 12], where it was argued that symmetries of the form eq. (1) emerge on very general grounds in the low-energy description of 2-dimensional systems, as a general consequence of pseudo-particle/vortex duality amongst the charge carriers in the low-energy effective theory relevant to low-temperature transport measurements.

In particular, ref. [10] showed the existence of two equivalence classes of systems, with $\Gamma_{0}(2)$ emerging as the symmetry relevant for systems related to fermionic 
charge carriers by a symmetry transformation and $\Gamma_{\theta}$ emerging as the symmetry relevant for systems related to bosonic charge carriers by a symmetry transformation. Indeed, these two subgroups are related by a conjugation operation which is equivalent to attaching one unit of statistical gauge field flux to the relevant pseudo-particles, thereby converting fermions to bosons and vice versa.

The power of the modular group was further realised in [13] where it was shown that the main experimental evidence for the Law of Corresponding states could be derived purely as a consequence of the emergent symmetries. Besides determining the positions of the possible Hall plateaux as low-temperature fixed points, the symmetries also govern how transitions can be made between quantum Hall plateaux as the magnetic field $B$ or the carrier density $n$ is varied. In particular, the spectacular semi-circle law for $B$-induced transitions between quantum Hall plateaux in the low-temperature limit can be derived assuming only that the flow through the conductivity plane as temperature is varied should be invariant under $\Gamma_{0}(2)$ symmetry (acting as in (1)) together with symmetry under particle-hole interchange. The symmetry also implies that the critical points along these semicircles occur at universal values of the conductivity for integer transitions at $\sigma=(k+i) / 2$, in units where $e^{2} / h=1$, where $k$ is an integer. Although many such points have been observed in transitions $\sigma: k \rightarrow k+1$ 14], spin effects can modify the precise position of the critical points [15, 16].

It is the great generality of these arguments which suggests seeking their test using the new Quantum Hall phenomena recently found in graphene. Graphene is unusual in that there is an extra twist in the statistics caused by a Berry phase of $\pi$ 17] when particles for each of the two low-energy bands are circulated around the point of band contact of the other low-energy band. We can realise this extra phase in the relative statistics of excitations in the two bands in terms of weakly interacting pseudo-particles carrying one extra unit of flux of a statistical gauge field - this flux would conjugate the relevant symmetry group from the usual $\Gamma_{0}(2)$ to $\Gamma_{\theta}$, as in 10], leading us to use the latter group to understand the graphene experiments in [1, 2]. This suggests that a hierarchical structure of quantum Hall plateaux, related by $\Gamma_{\theta}$, should eventually be found in graphene, given samples with sufficiently high mobility.

Another complication for graphene at moderate magnetic fields is the spin degeneracy of the conduction electrons due to the small Zeeman splitting between electron spin states. We provide details about how such degeneracies alter the implications of modular symmetries elsewhere [18], whose arguments lead us to consider the modular group $\Gamma_{\theta}$ acting on $\sigma / 2$, with the factor of $1 / 2$ arising because of the spin degeneracy. Note that the symmetry therefore includes the transformation $\sigma \rightarrow \sigma+4$, which in a fermionic system has the interpretation of Landau level addition with four-fold degeneracy, such as would be the usual interpretation for this transformation in graphene.

The consequences of $\Gamma_{\theta}$ symmetry for the temperature flow of the conductivities were given in [10], the resulting temperature flow diagram is reproduced here as Figure 1. The predictions for graphene differ from those given in [10], however, in two ways. First, because of the spin degeneracy Figure 1 here should be regarded as plotting the real and imaginary parts of $\sigma / 2$ rather than $\sigma$.

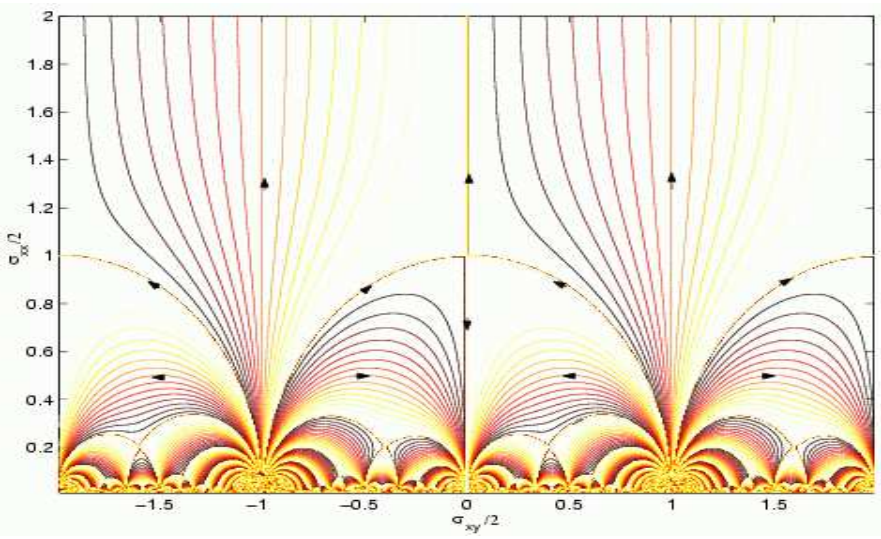

Figure 1: $\Gamma_{\theta}$ temperature flow (colour online).

Second, the direction of the temperature flow in the conductivity plane given in Figure 1 is that given in [10], which was drawn to represent the direction of decreasing temperature assuming that the underlying system at zero magnetic field $\left(\sigma_{x y}=0\right)$ describes a superconductor/insulator system, with $\sigma_{x x} \rightarrow i \infty$ or 0 as $T \rightarrow 0$. (Notice the universal fixed point which is predicted at $\sigma_{x x}=e^{2} / h$ describing the superconductor-insulator transition [19].) However, graphene is a semi-metal and so for it one instead expects the conductivity at vanishing magnetic field to decrease away from $\sigma_{x x}=i \infty$ as $T$ is lowered. Thus the flow direction shown in Figure 1 should be taken to represent increasing temperature for graphene.

We may immediately draw a number of robust consequences concerning the allowed Hall plateaux for graphene systems, whose validity relies on the absence of impurities and/or defects which can destroy the particlevortex symmetry on which $\Gamma_{\theta}$ is based.

- Integer Quantum Hall Plateaux: The allowed Hall plateaux correspond to the attractive fixed points of the flow in the limit that $T \rightarrow 0$, all of which lie along the real axis (for which $\sigma_{x x}=0$. Keeping in mind the two-fold degeneracy and the flow direction described above, inspection of the figure (with the arrows reversed) shows that all such integer fixed points occur at $\sigma_{x y} / 2=2 k+1$, for integer $k$. This implies integer Quantum Hall plateaux at $\sigma_{x y}=4 k+2$, as is seen for single-layer graphene systems [1, 2] in intermediate magnetic fields.

- Fractional Quantum Hall Plateaux: The complete set of attractive fixed points as $T \rightarrow 0$ occur at $\sigma_{x y}=2 p / q$, 
with $p$ and $q$ being relatively prime odd integers. For higher mobility single-layer graphene samples we therefore predict the existence of a hierarchy of fractional Quantum Hall plateaux at precisely these fractional values (and no others), at least for the same range of magnetic fields for which the above integer effect is seen.

But modular symmetry gives more. Besides identifying the low-temperature fixed points, taken together with particle-hole symmetry $\Gamma_{\theta}$ invariance also dictates the detailed form of the transitions which can take place between the allowed plateaux as either magnetic field, $B$, or carrier density, $n$, are varied. These two symmetries can do so because they suffice to guarantee [13] that the caustics of Figure 1 - i.e. the straight lines and semicircles on which those fixed points lie which are not on the real axis - are bona fide temperature flow lines. (By contrast, the detailed shape of the remaining flow lines shown in Figure 1 are not similarly dictated purely by the symmetries). If one compares a family of temperature flow curves for different $B$ (or $n$ ), and connects the points which correspond to the same value of $T$, then one finds the resulting curves are inevitably pushed to lie along one of the caustics in the $\sigma$ plane as $T \rightarrow 0$.

This observation leads to the following further predictions:

- Selection Rule: A consequence of the above arguments is that any low-temperature transition between a pair of plateaux (with varying $B$ or $n$ ) must be the image under $\Gamma_{\theta}$ of the basic semicircle running from -1 to 1 of Figure 1. This implies in particular that transitions are not possible between arbitrary pairs of Hall plateaux. Instead, the symmetry allows transitions between two plateaux, $\sigma=2 p / q$ and $\sigma=2 p^{\prime} / q^{\prime}$, only when

$$
\left|p q^{\prime}-p^{\prime} q\right|=2 \text {, }
$$

which is compatible with the previous observation that $p, p^{\prime}, q$ and $q^{\prime}$ are all odd for attractive fixed points. A selection rule of this type for quantum Hall transitions due to modular symmetry was first derived in [14] and (2) is the rule for graphene.

- Universal Transition Points I: Given that the transition between $\sigma=-2$ and $\sigma=2$ must move along the semicircle of radius 2 centered at the origin, we have a universal prediction that the value attained by $\rho_{x x}$ in the absence of Hall conductivity, $\sigma_{x y}=0$, corresponds to the point $\sigma=2 i$ : that is, $\rho_{x x}=1 / 2$ (in units where $e^{2} / h=1$ ). Indeed, the experimental data in [1] show a peak in $\rho_{x x}$, when $\sigma_{x y}=0$, very close to $12.9 k \Omega=h / 2 e^{2}$. Modular symmetry implies that this point should be a fixed point corresponding to the phase transition which occurs as the system bifurcates from reaching $\sigma=-2$ to $\sigma=2$ in the low-temperature limit. This is the direct analogue of the second order phase transition at $\sigma=k+i / 2$ in ordinary monolayer semiconductor samples [20]. Notice that although these predictions qualitatively agree with some recent calculations, which also predict a universal value for $\sigma_{x x}$ when $\sigma_{x y}=0$ [21], they differ in the universal value which is predicted.

- Universal Transition Points II: Since all transition curves are the image of the basic one under $\Gamma_{\theta}$, we can predict exactly where the bifurcation points arise for all other transition curves. Since the group element required to obtain the transition $\sigma / 2: p / q \rightarrow p^{\prime} / q^{\prime}$ from the basic one between $\sigma / 2:-1 \rightarrow 1$, has $a=\left(p^{\prime}-p\right) / 2$, $b=\left(p^{\prime}+p\right) / 2, c=\left(q^{\prime}-q\right) / 2$, and $d=\left(q^{\prime}+q\right) / 2$, it follows that the critical point at $\sigma / 2=i$ in the in the -1 to 1 transition is mapped to $\sigma / 2=\left(p q+p^{\prime} q^{\prime}+2 i\right) /\left(q^{2}+q^{\prime 2}\right)$ in the $p / q$ to $p^{\prime} / q^{\prime}$ transition, and so

$$
\sigma=\frac{2\left(p q+p^{\prime} q^{\prime}\right)+4 i}{q^{2}+q^{\prime 2}} .
$$

This is therefore the critical bifurcation point for the transition $\sigma: 2 p / q \rightarrow 2 p^{\prime} / q^{\prime}$.

- Semi-circle Law: Besides predicting the positions of the critical points, as argued above $\Gamma_{\theta}$ symmetry determines the entire shape of the transition curve traced out within the conductivity plane as $B$ or $n$ varies at low temperature, and predicts it to follow a precisely semi-circular trajectory [13]. With this paper we hope to encourage experimentalists to check this semi-circular prediction.

As a preliminary test we have examined the experimental data in [1] (the data in [2] are not presented in a way that allowed us to perform the analysis on that experiment), a figure of which is reproduced here as Figure 2. This figure plots the Ohmic resistivity, $\rho_{x x}$ and the Hall conductivity, $\sigma_{x y}$, as reported by [1] as a function of carrier density, $n$. Because we were unable to infer directly from this the trajectory taken in the complex $\sigma$ plane with sufficient accuracy, we instead chose to directly compare the curves in this figure with a specific parameterization, $\sigma(n)$ (related to that in [22], whose detailed form does not follow purely on symmetry grounds). By assuming semi-circular trajectories for the transitions $\sigma:-10 \rightarrow-6 \rightarrow-2 \rightarrow 2 \rightarrow 6 \rightarrow 10$ we obtain the blue curves in Figure 2, overlaid on the experimental curves. To obtain these curves we use our assumed parameterization to fit $\rho_{x x}$ for the $\sigma:-2 \rightarrow 2$ transition by eye, constrained by the fact that it should be symmetric about the vertical axis and that the maximum is not a free parameter but must lie at $12.9 k \Omega$. We then derive $\sigma_{x x}$ for this transition by assuming that it is semi-circle in the $\sigma$-plane. This semi-circle is then mapped to the other transitions by using $\sigma \rightarrow \sigma \pm 4$, and $\rho_{x x}$ is calculated for these transitions. Once $\rho_{x x}$ for the $-2 \rightarrow 2$ transition is determined all of the other transitions are fit with only one free parameter, namely the horizontal shift necessary to line up the $\sigma_{x y}$ curve with the experimental data. Although our plots use a specific parameterization we believe this is not likely to be essential for the 
present purposes - what matters is that the blue curves are semi-circles in the $\sigma$-plane.

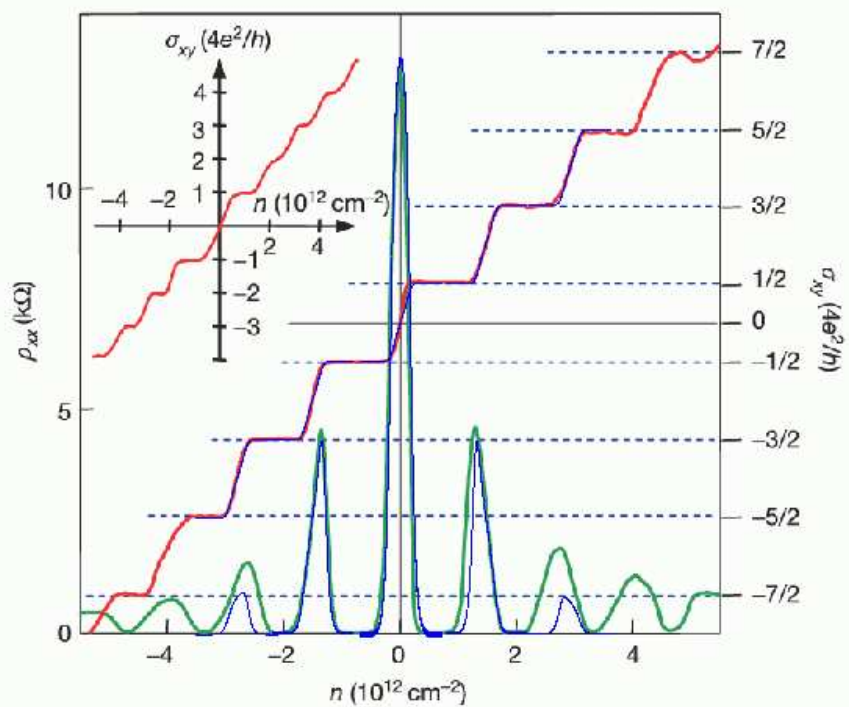

Figure 2: A semicircle (solid blue line) overlaid on the data of [1] (colour online).

The visual agreement between the semi-circle curves and the experimental data seems rather good for $-6 \rightarrow$ $-2 \rightarrow 2 \rightarrow 6$, though the fit to $\rho_{x x}$ is poorer for $2 \rightarrow 6$ than for $-2 \rightarrow-6$, worse again for the higher plateaux. For the transition $\sigma: 4 k-2 \rightarrow 4 k+2$ a semicircle implies that the peak in $\rho_{x x}$ should lie at $\rho_{x x}^{\max }=$ $\left(2\left|4 k^{2}-1\right|\right)^{-1} k \Omega$, and relative to this the $\pm 10 \rightarrow \pm 6$, (which a semi-circle predicts should be at $\rho_{x x}=0.85 \mathrm{k} \Omega$ ) are clearly too high. This indicates a breakdown of either particle-hole symmetry or the particle-vortex duality underlying the $\Gamma_{\theta}$ symmetry.

At still higher fields, over $40 T$, the picture changes and more plateaux are visible, which are interpreted as being due to lifting the spin degeneracy [23]. We do not know how to interpret these high-field data in terms of modular symmetries, or whether such an interpretation is possible. These data do not appear to be compatible with $\Gamma_{\theta}$ which cannot exhibit stable plateaux at both even and odd integers (with pseudo-particles of charge $e$ and $\left.e^{2} / h=1\right)$ - perhaps they represent a transition between two regimes. It would be very useful to have data exploring more of the $\sigma$-plane for a range of values of $B$ and $n$.

The hypothesis that $\Gamma_{\theta}$ is an underlying symmetry of the quantum Hall effect in single-layer graphene (for intermediate magnetic field values) clearly makes many robust predictions. If borne out by further measurements, the existence of the emergent $\Gamma_{\theta}$ symmetry has implications for the microscopic physics which is responsible. It does so because of what it says about the properties of the pseudo-particle charge carriers which appear in the low- energy effective description of low-temperature transport properties. In particular, the appearance of the 'bosonic' group $\Gamma_{\theta}$ provides an additional observational implication of the Berry phase, perhaps pointing to a composite Fermion picture 24] for graphene which attaches an odd number of statistical gauge field flux quanta to each pseudo-particle rather than an even number.

Acknowledgments: BD thanks the Perimeter Institute, for hospitality during this investigation.

[1] K.S. Novoselov et al, Nature 438197 (2005)

[2] Y. Zhang, Yan-Wen Tang, H.L. Stormer and P. Kim, Nature 438201 (2005)

[3] A. Shapere and F. Wilczek, Nuc. Phys. B320 669 (1989)

[4] C.A. Lütken and G.G. Ross, Phys. Rev. B45, 11837 (1992)

[5] C.A. Lütken and G.G. Ross, Phys. Rev. B48, 2500 (1993)

[6] C.A. Lütken, J. Phys. A26 L811 (1993); Nuc. Phys. B396 670 (1993); C.P. Burgess and C.A. Lutken, Nuc. Phys. 500 (1997) 367, [cond-mat/9611118]; C.P. Burgess and C.A. Lutken, Phys. Let. B451 365 (1999), [cond-mat/9812396].

[7] D-H. Lee, S. Kivelson and S-C. Zhang, Phys. Lett. 68, 2386 (1992); S. Kivelson, D-H. Lee and S-C. Zhang, Phys. Rev. B46, 2223 (1992)

[8] J.K Jain, S.A. Kivelson and N. Trivedi, Phys. Rev. Lett. 64 (1990) 1927; J.K. Jain and V.J. Goldman, Phys. Rev. B45 1255 (1992)

[9] D. Shahar et al., Science 274, 589 (1996).

[10] C.P. Burgess and B.P. Dolan, Phys. Rev. B63 155309 (2001), [hep-th/0010246]

[11] C.P. Burgess and B.P. Dolan, Phys. Rev. B65 155323 (2002), [cond-mat/0105621]

[12] E. Witten, in Shifman, M. (ed.) et al.: From fields to strings, vol. 2, 1173-1200, hep-th/0307041.

[13] C.P. Burgess, Rim Dib and B.P. Dolan, Phys. Rev. B62 15359 (2000), [cond-mat/9911476];

[14] B.P. Dolan, J. Phys. A32 L243 (1999), [cond-mat/9805171]

[15] B.P. Dolan, Phys. Rev. B62, 10278 (2000), [cond-mat/0002228]

[16] C.F. Huang et al, [cond-mat/0609310]

[17] T. Ando, T. Nakaishi and R. Saito, J. Phys. Soc. Jpn. 67 2857 (1998); G.P. Mikitik and Y.V. Sharlai, Phys. Rev. Lett. 822147 (1999)

[18] C.P. Burgess and B. P. Dolan, in preparation.

[19] M.P.A. Fisher, Phys. Rev. Lett. 65, 923 (1990)

[20] D. Shahar et al., Phys. Rev. Lett. 79, 479 (1997)

[21] V.P. Gusynin and S.G. Sharapov, Phys. Rev. Lett. 95 146801 (2005)

[22] B.P. Dolan, Nuc. Phys. B554 [FS] 487 (1999), [cond-mat/9809294]

[23] Y. Zhang et al, Phys. Rev. Lett. 96136806 (2006)

[24] J.K. Jain, Phys. Rev. Lett. 63 (1989) 199; Phys. Rev. B41 7653 (1990) 